\title{
Cáscara de cacao fuente de polifenoles y fibra: simulación de una planta piloto para su extracción
}

\section{Cocoa husk source of polyphenol and fiber: simulation of a pilot plant for their extraction}

\section{Fonte de shell de cacau de polifenóis e fibras: simulação de uma planta piloto para a remoção}

\author{
Arley René Villamizar-Jaimes ${ }^{1}$, Luis Javier López-Giraldo²
}

Forma de citar: A.R. Villamizar-Jaimes y L.J. López-Giraldo, "Cáscara de cacao fuente de polifenoles y fibra: simulación de una planta piloto para su extracción”, Respuestas, vol. 22, no. 1, pp. 75-83, 2017.

\author{
Recibido: \\ Agosto 9 de 2016 \\ Aceptado: \\ Noviembre 24 de \\ 2016
}

${ }^{1}$ Químico

arleyvil@uis.edu.co Orcid:000-0002-6042-7618

Universidad Santo Tomás-

Universidad Industrial de Santander

Bucaramanga- Colombia.

${ }^{2}$ Doctor en Química, Bioquímica

y Ciencia de Alimentos, ljlopez@uis.edu.co

Orcid: 0000-0001-9267-7016

Grupo de Investigación en

Ciencia y Tecnología de

Alimentos- CICTA

AgroBiotech Research Center-

$\mathrm{ABC}$, Docente

Universidad Industrial de

Santander

Bucaramanga-Colombia

\section{Resumen}

En el municipio de San Vicente de Chucurí, Santander, se produce aproximadamente el $11,8 \%$ del cacao de Colombia. Asociado con la producción de los granos de cacao se producen de $74-86 \%$ de residuos durante el proceso de beneficio, lo que representaría entre 4715-5480 toneladas al año. Esta situación genera problemas ambientales y fitosanitarios que se deben atender en el futuro inmediato. Es así que en este trabajo se estudia el aprovechamiento de los residuos de cacao (cáscara) del material clon CCN-51 para la extracción de polifenoles y fibra total. Desde el punto de vista metodológico, se desarrollaron dos etapas; en la primera se caracterizaron las cáscaras de cacao evaluando el contenido de polifenoles totales (método de Folin-Ciocalteu) y fibra dietaria total (método enzimático-gravimétrico); en lo que respecta a la segunda etapa, se realizó la simulación (software SuperPro Designer ${ }^{\circledR}$ v.9.0 académica) de una planta extractora usando como información de entrada al simulador los resultados obtenidos en la primera etapa. La simulación se desarrolló tomando como base de cálculo el 32,6\% de la producción del municipio y los resultados fueron: el contenido de polifenoles totales y fibra dietaria total para el clon CCN-51 fueron respectivamente $61 \mathrm{mgEAG} / \mathrm{g}$ (expresados en peso seco) y $56,80 \%$. En lo que respecta a la simulación, se propuso un diagrama de proceso en que se incluyeron las etapas de preparación, extracción, concentración de polifenoles y fibra total. Con esta configuración fue posible obtener $8,6 \mathrm{kgEAG} / \mathrm{h}$ de polifenoles totales y $80,3 \mathrm{~kg} / \mathrm{h}$ de fibra total presentes en la torta de cáscara de cacao.

Palabras clave: Cáscara de cacao, fibra dietaria total, polifenoles, simulación. 
No. 1

Enero - Junio 2017 ISSN 0122-820X E-ISSN 2422-5053

a simulation (SuperPro Designer ${ }^{\circledR}$ software, academic version 9.0) of an extraction plant was performed, using the values of obtained experimentally in the first stage as input data of the simulator. The simulation was developed on the basis of calculating the $32.6 \%$ of production in the municipality of San Vicente and the results obtained were as follows: the content of total polyphenols and total dietary fiber for clone CCN-51 were respectively $61 \mathrm{mgEGA} / \mathrm{gdm}$ y $56,80 \%$. Regarding the simulation, a process diagram was proposed in which the stages of preparation, extraction and concentration of polyphenols and total dietary fiber were included. With this configuration, it was possible to obtain $8,7 \mathrm{kgEGA} / \mathrm{h}$ total polyphenols and $80,3 \mathrm{~kg} / \mathrm{h}$ total dietary fiber present in the cocoa husk mass.

Keywords: Cocoa Husk, Total dietary fiber, Polyphenol, Simulation.

\section{Resumo}

No município de San Vicente de Chucurí, Santander, que ocorre aproximadamente 11,8\% de cacau na Colômbia. Associado com a produção de grãos de cacau são produzidos $74-86 \%$ dos resíduos durante o processo de beneficiamento, o que representaria entre 4715-5480 toneladas por ano. Isso cria problemas ambientais e fitossanitárias que devem ser abordadas no futuro imediato. Assim, neste estudo é estudada a utilização de cacau (escudo) de material de resíduos CCN-51 clone para a extracção de polifenóis e fibra total. Do ponto de vista metodológico, duas etapas foram desenvolvidas; no primeiro as conchas de cacau foram caracterizadas através da avaliação do teor de polifenóis total (de Folin-Ciocalteu) e de fibra dietética total (método de enzima-gravimétrica); com respeito ao segundo ponto, foi realizada a simulação (software Designer SuperPro @v.9.0 académico) utilizando uma instalação de extracção como entrada para o simulador de os resultados obtidos no primeiro passo. A simulação foi desenvolvido cálculo baseado $32,6 \%$ da produção do município e os resultados foram: o conteúdo de polifenóis totais e fibra total para o clone CCN-51 foram, respectivamente, $61 \mathrm{mgEAG}$ dietética / g (peso seco expresso ) e $56,80 \%$. No que diz respeito à simulação, um diagrama de processo em que incluiu propôs as fases da preparação, extracção, concentração de polifenóis e fibra total. Com esta configuração foi possível obter $8,6 \mathrm{kgEAG} / \mathrm{h}$ e de polifenóis totais 80,3 $\mathrm{kg} / \mathrm{h}$ de fibra total presente no bolo de casca de cacau.

Palavras-chave: casca de cacau, fibra dietética total, polifenóis, simulação.

\section{Introducción}

El clon de cacao CCN 51 (Colección Castro 76 Naranjal) es originario de Ecuador en donde fue obtenido de cepas de cacao de Iquitos, criollo y amelonado por Homero Castro en 1965, es un clon resistente a diferentes plagas que lo han hecho un cacao de alta productividad y resistencia. Este clon ha sido también adoptado en países como Perú y Colombia en donde ha tenido alta promoción y desarrollo debido a su alta productividad que oscila entre 2000 a 3000 kilos por hectárea en condiciones óptimas de producción [1]. Además de la alta productividad y resistencia, Pallares - Pallares et al. [2] concluyen recientemente que bajo condiciones controladas de fermentación, es posible obtener a partir de este material notas odoríficas muy agradables que son apetecidas por mercados especializados.

Una gran variedad de clones de cacao se encuentra presentes en todas las veredas del municipio de San Vicente de Chucurí, $\mathrm{y}$ han sido recomendados por entidades como FEDECACAO por sus características productivas; se tiene que el clon $\mathrm{CCN} 51$ cuenta con un $25 \%$ de participación, el ICS 
60 con un 14\% y el ICS 95 con un $25 \%$, de la producción del departamento [3].

Las cáscaras de cacao se obtienen cuando los granos son separados de la vaina. El grano de cacao es utilizado principalmente para la fabricación del chocolate; sin embargo, en el beneficio y procesamiento del grano se generan residuos, tales como cáscaras, cascarillas y efluentes de fermentación, que representan hasta el 74-86\% (en peso) de la mazorca [4]. Las cáscaras de cacao son ricas en carbohidratos, fibra, proteínas, pectina y compuestos bioactivos (por ejemplo, los polifenoles y carotenoides) [5]. El alto porcentaje de residuos de cacao, hace de estos un problema ambiental y fitosanitario en las plantaciones de cacao, pero teniendo en cuenta sus niveles nutricionales estos residuos pueden ser empleados para obtener productos con un alto valor agregado; entre los que se destacan los polifenoles y la fibra que se posicionan muy bien en los mercados actuales [6].

Entre las opciones para la valorización de la cáscara de cacao, se han abordado diferentes puntos de vista, en los cuales se estudian los contenidos de fibra y la cantidad de polifenoles totales. Los resultados de Flórez y Jerez [7], muestran que las cáscaras de cacao tienen un contenido de fibra dietaria total de aproximadamente $60 \%$ en peso.

En lo que se refiere a la capacidad antioxidante, el contenido de polifenoles totales y su respectivo perfil; los resultados de Toro et al. [8], evidencian que es posible obtener extractos de polifenoles a partir de la cáscara de cacao con un contenido de polifenoles totales que varía entre 60 - 91mgEAG/g, para cinco materiales evaluados. En cuanto al perfil de polifenoles, usando HPLC-ESI-MS, los resultados muestran que en los extractos de la cáscara de cacao de diferentes genotipos contienen (+)-catequina, (-)-epicatequina, procianidinas B1 y B2, ácido gálico, galocatequina, clovamida y disacáridos. Así mismo, en el mismo trabajo se determinó la capacidad antioxidante empleando el método ORAC y los resultados para los extractos de los cinco materiales estudiados estuvieron entre $1528,2 \pm 303,9$ y $2538,06 \pm 532,81 \mathrm{~mol}$ $\mathrm{TE} /$ g. Estos resultados muestran que es posible obtener productos de mayor valor agregado a partir de este tipo de residuos y que para la industria de compuestos funcionales esta es una alternativa de obtener metabolitos secundarios, dada su incidencia en el bienestar y salud de posibles consumidores [9].

Sin embargo, la propuesta de obtención de este tipo de productos debe iniciar con la simulación del proceso. En ese sentido, la simulación de procesos agroindustriales empleando software especializado, es de gran importancia en el campo de la agroindustria ya que permiten diseñar, identificar fallas y plantear posibles cambios en la línea productiva. Se ha demostrado que el software SuperPro Designer ${ }^{\circledR}$ v.9.0 académica, es una herramienta de gran utilidad para el análisis de procesos en la industria [10].

En el proceso preliminar descrito por Villamizar y Barragan [11] para la extracción de polifenoles empleando cáscara de cacao, las etapas de pretratamiento y concentración requieren grandes cantidades de energía por lo cual es necesario implementar una integración energética o una alternativa para disminuir los costos relacionados con servicios públicos del proceso. Por otra parte, la torta de cáscara de cacao que se produjo en dicho proceso, es una fuente de fibra que puede ser valorizada, repercutiendo así en la economía del proceso diseñado.

Con base en lo anterior, el alcance u objetivo de este trabajo es simular un proceso de extracción de polifenoles y fibra dietaría total, empleando como materia prima las cáscaras de cacao y utilizando tecnología que genere un impacto positivo a nivel ambiental. Esto
Enero - Junio 2017 ISSN 0122-820X E-ISSN 2422-5053 PP: $75-83$ 
No. 1

Enero - Junio 2017 ISSN 0122-820X E-ISSN 2422-5053

PP: $75-83$ último acorde con la tendencia tecnológica de producción más limpia y desarrollo sostenible.

\section{Materiales y métodos}

\subsection{Materiales}

Las cáscaras de cacao frescas del material CCN-51 fueron recolectadas de fincas de San Vicente de Chucurí-Santander. El análisis bromatológico incluyó la determinación de humedad AOAC 931.04, ceniza AOAC 972.15, grasa AOAC 963.15, proteína AOAC 970.22, fibra cruda AOAC 930.20.

\subsection{Preparación del extracto}

Las cáscaras de cacao fueron lavadas manualmente, cortadas y tratadas térmicamente para inhibir la enzima polifenol oxidasa, siguiendo el protocolo optimizado por Godoy [12] que garantiza la inhibición de la enzima PPO y contribuye a una mayor recuperación de monómeros, en específico, la (-)-epicatequina.

La cáscara fue secada a $50{ }^{\circ} \mathrm{C}$ y el material fue molido para obtener la harina de cáscara de cacao. Las condiciones óptimas para la extracción son $60{ }^{\circ} \mathrm{C}$, relación etanol:agua $50: 50 \mathrm{v} / \mathrm{v}$, relación cáscara:solvente 1:60 $\mathrm{p} / \mathrm{v}$ y $\mathrm{pH} 5$ [12]. Estos autores comprobaron que aplicando las condiciones de extracción enunciadas en el párrafo anterior, se consigue maximizar el contenido de polifenoles totales extraíbles desde cáscaras de cacao de 5 materiales diferentes.

\subsection{Determinación del contenido total de polifenoles}

El contenido de polifenoles totales en el extracto de la cáscara de cacao se calculó a partir de la capacidad de reducción del reactivo Folin-Ciocalteu. La absorbancia fue medida a $765 \mathrm{~nm}$ usando un espectrofotómetro (MULTISKAN GO ThermoScientific). Los resultados fueron expresados en $\mathrm{mg}$ equivalentes de ácido gálico por gramos de cáscara de cacao seca (mg EGA/g).

\subsection{Análisis de fibra dietaría total}

La determinación de fibra dietaria total, insoluble y soluble se realizó empleando el método enzimático-gravimétrico basado en la AOAC 991.43. Las principales modificaciones hechas al procedimiento estandarizado fueron:

a. Proceso de filtrado: el papel filtro empleado fue papel filtro analítico en lugar de filtro celite, [13]

b. La enzima proteasa fue cambiada por la pepsina [7]

\subsection{Simulación del proceso}

La simulación se realizó con base en los resultados a escala laboratorio obtenidos de la caracterización de polifenoles y fibra dietaría total para la cáscara de cacao del material CCN-51.

El procedimiento se desarrolló en SuperPro Designer ${ }^{\circledR}$ v.9.0 académica. En una primera etapa se definieron las familias de compuestos que conforman la cáscara de cacao con base en la caracterización fisicoquímica experimental (Ver tabla I). Las moléculas simuladas son polifenoles, pectinas y fibra; representadas por ácido gálico, ácido D-galacturónico y celulosa respectivamente.

Así mismo, en la tabla II presentan las condiciones que se introducen en cada una de las unidades de interés del proceso simuladas.

Para la simulación se tuvo en cuenta las siguientes consideraciones:

a. El proceso tiene contemplado manejar una producción en continuo de aproximadamente $3400 \mathrm{~kg}$ /día de cáscaras del material CCN-51. El cual representa el $25 \%$ de la producción de cacao de San Vicente de Chucurí. 
b. El proceso de limpieza, inactivación de la polifenol oxidasa y secado de la cáscara de cacao se realiza en la finca, siguiendo el procedimiento descrito por Carreño et al [14].

c. Los principales componentes para la cáscara fueron agua (humedad), ácido gálico (polifenoles), ácido galacturonico (pectinas), celulosa (fibra) y matriz (hace referencia a la proteína, grasa, carbohidratos y minerales). d. La extracción de polifenoles se llevó a cabo como un proceso compuesto por tres etapas: a) Pretratamiento, b) Extracción y c) Recuperación.

e. Se consideró un flujo de etanol-agua de $1110 \mathrm{~kg} / \mathrm{h}$ para la extracción de 141,351 $\mathrm{kg} / \mathrm{h}$ de cáscara de cacao.

La figura 1 esquematiza del proceso de recuperación de polifenoles a partir de la cáscara de cacao, implementado en SuperPro Designer ${ }^{\circledR}$ v.9.0 académica.

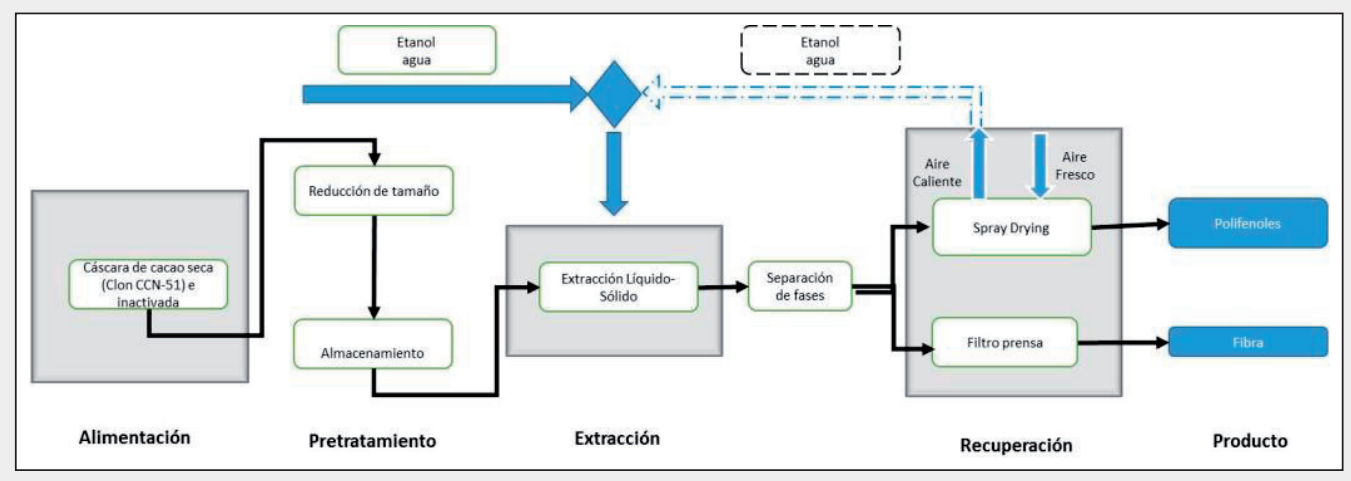

Figura 1. Esquema del proceso de extracción de polifenoles y fibra empleando $3400 \mathrm{~kg} / \mathrm{día}$ de cáscaras de cacao CCN51.

Fuente: Autores.

\section{Resultados y análisis}

Las características fisicoquímicas de la cáscara de cacao del material CCN51 se muestran en la tabla I.

Tabla I. Características bromatológicas y fisicoquímicas de la cáscara de cacao CCN51.

\begin{tabular}{|c|c|c|}
\hline Molécula Simulada & Característica fisicoquímica & Resultado [\%] \\
\hline Agua & Humedad & $6,7 \pm 0,3$ \\
\hline Celulosa & Fibra dietaría total & $56,8 \pm 4,0$ \\
\hline Ácido gálico & Polifenoles totales & $6,1 \pm 1,8$ \\
\hline Ácido D-galacturonico & Pectinas & $1,0 \pm 0,1$ \\
\hline \multirow{2}{*}{ Matriz } & Ceniza & $11,4 \pm 0,1$ \\
& Grasa & $0,7 \pm 0,1$ \\
& Proteína & $6,3 \pm 0,1$ \\
& Azúcares & $11,0 \pm 0,1$ \\
\cline { 2 - 3 } & Total & $29,4 \pm 0,1$ \\
\hline
\end{tabular}

Fuente: Autores

Con base en la figura 1 se construyó el diagrama de flujo del proceso usando el software SuperPro Designer ${ }^{\circledR} \quad$ v.9.0 académica, que provee los balances de masa, energía, el dimensionamiento de los equipos y la potencia requerida. En la figura 2, se aprecian cada una de las etapas del proceso simuladas, cuyos parámetros de entrada y los resultados obtenidos de la simulación en la extracción de polifenoles totales (PT) y fibra dietaria (FD) más sobresalientes se muestran en la tabla II. 


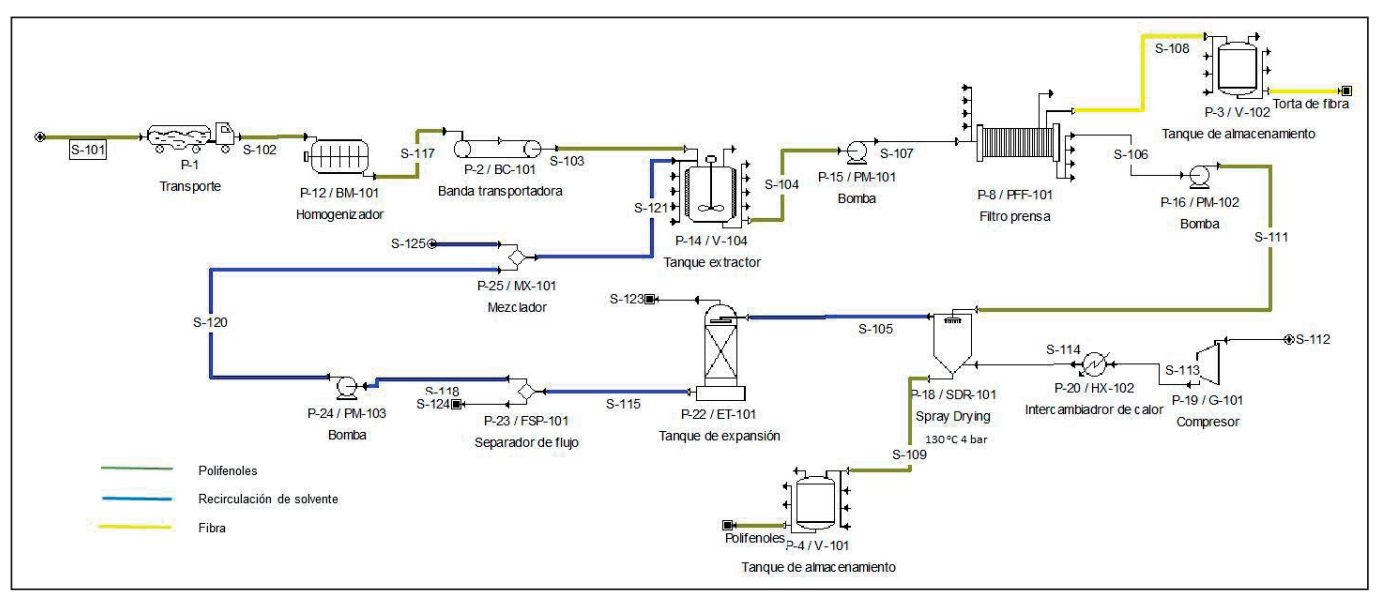

Figura 2. Diagrama de proceso de extracción de FD y PT simulado empleando el software SuperPro Designer ${ }^{\circledR}$ v.9.0 académica Fuente: Autores.

Tabla II. Corrientes asociadas con la etapa de extracción de PT y concentración de FD empleando cáscara de cacao del clon CCN51 con recirculación de solvente usando el software SuperPro Designer ${ }^{\circledR}$ v.9.0 académica

\begin{tabular}{|c|c|c|c|c|c|c|c|c|c|}
\hline \multirow{2}{*}{$\begin{array}{l}\text { Componente } \\
(\mathrm{kg} / \mathrm{h})\end{array}$} & \multicolumn{3}{|c|}{ Extracción } & \multicolumn{2}{|c|}{ Recuperación de FD } & \multicolumn{4}{|c|}{ Recuperación PT } \\
\hline & $\begin{array}{l}\text { S-103 } \\
\text { (e) }\end{array}$ & $\begin{array}{l}\mathrm{S}-121 \\
\text { (e) }\end{array}$ & $\begin{array}{l}\mathrm{S}-104 \\
(\mathrm{~s})\end{array}$ & $\begin{array}{l}\text { S-107 } \\
\text { (e) }\end{array}$ & $\begin{array}{l}\text { FD } \\
(\mathrm{s})\end{array}$ & S-111 (e) & S-114 (e) & S-105 (s) & $\begin{array}{l}\text { PT } \\
(\mathrm{s})\end{array}$ \\
\hline Nitrógeno & -- & -- & -- & -- & -- & -- & 22326,47 & 22326,47 & -- \\
\hline Oxígeno & -- & -- & -- & -- & -- & -- & 6677,88 & 6677,88 & -- \\
\hline Alcohol etílico & -- & 4339,06 & 4339,06 & 4339,06 & 10,17 & 4328,88 & -- & 4328,88 & -- \\
\hline Fibra & 80,29 & -- & 80,29 & 80,29 & 80,29 & -- & -- & -- & -- \\
\hline Ácido Galacturónico & 1,46 & -- & 1,46 & 1,46 & 1,46 & -- & -- & -- & -- \\
\hline Acido Gálico & 8,62 & -- & 8,62 & 8,62 & 0,02 & 8,60 & -- & -- & 8,60 \\
\hline Matriz & 41,55 & -- & 41,55 & 41,55 & 41,55 & -- & -- & -- & -- \\
\hline Agua & 9,43 & 1486,06 & 1495,49 & 1495,49 & 3,51 & 1491,99 & -- & 1491,99 & -- \\
\hline Presión $(\mathrm{kPa})$ & 101,33 & 101,33 & 101,33 & 151,33 & 151,33 & 171,32 & 607,95 & 101,32 & 101,32 \\
\hline Temperatura $(\mathrm{K})$ & 298,15 & 334,62 & 313,15 & 336,30 & 336,30 & 336,31 & 423,15 & 343,15 & 298,15 \\
\hline $\begin{array}{l}\text { Entalpía } \\
(\mathrm{kcal} / \mathrm{h})\end{array}$ & 965,7 & 247534,09 & 256738,14 & 256772,66 & 2385,41 & 254397,69 & 1060782,99 & 775355,82 & 22,73 \\
\hline
\end{tabular}

(e) entrada, (s) salida

Fuente: Autores.

En la tabla III, se aprecia que la energía necesaria para el desarrollo del proceso sería 2953,1 kW-h/h, de los cuales el 98,5\% corresponde a la etapa de recuperación de polifenoles, concluyéndose así que esta etapa es la más demandante en energía.

Tabla III. Requerimientos energéticos y de solvente por etapas usando el software SuperPro Designer ${ }^{\circledR}$ v.9.0 académica

\begin{tabular}{|c|c|c|c|c|}
\hline \multirow{2}{*}{ SECCIÓN } & \multicolumn{2}{|c|}{ POTENCIA [kW-h/h] } & \multicolumn{2}{|c|}{ SOLVENTE (kg/h) } \\
\hline & Con reciclo & Sin reciclo & Sin reciclo & Con reciclo \\
\hline Pretratamiento & 33,8 & 33,8 & --- & --- \\
\hline Extracción & 9,6 & 9,6 & 5825,1 & 1110,0 \\
\hline Recuperación & 2909,6 & 2908,9 & --- & --- \\
\hline TOTAL REQUERIDA & 2953,1 & 2952,3 & --- & --- \\
\hline
\end{tabular}

Fuente: Autores.

Teniendo en cuenta este criterio, se deberá a futuro pensar en la integración de corrientes de proceso con el fin de disminuir la demanda energética. Por ejemplo, el fluido de servicio que sale del intercambiador P-20 podría integrarse como fluido caliente para el proceso de extracción. Otra opción es que la corriente S-123 (aire caliente a $70^{\circ} \mathrm{C}$ ) se envíe nuevamente al proceso mezclándolo con el aire fresco que sale del compresor P-19. 
Con relación a los productos (fibra y polifenoles) se observa que por cada 141,351 $\mathrm{kg} / \mathrm{h}$ de cáscara se pueden obtener 8,60 $\mathrm{kgEGA} / \mathrm{h}$ de polifenoles y $80,29 \mathrm{~kg} / \mathrm{h}$ torta de fibra, aprovechándose así un $62.9 \%$ del residuo de cacao.

A partir de la tabla III, se puede inferir que al complementar el proceso con la recirculación de solventes el costo energético es similar al sin recirculación. La gran cantidad de energía en el proceso se debe al alto flujo de aire que debe alimentarse al compresor y al intercambiador de calor $(29102,38 \mathrm{~kg} / \mathrm{h})$ para garantizar la alta remoción $(>95 \%)$ de solvente en el producto final (polifenoles totales).

En lo que se refiere al uso de solvente, se aprecia que la corriente de (etanol:agua) fresco en el proceso sin recirculación es de 5825,10 $\mathrm{kg} / \mathrm{h}$ y que en el caso con recirculación es de $1110 \mathrm{~kg} / \mathrm{h}$, proviene del lazo de recirculación S-120. El ahorro en solvente por recirculación es de $81 \%$.

Para este trabajo se contempló la alternativa de recirculación de solvente, dirigida a reducir los costos de proceso y reducir el volumen de residuos químicos que se generan del mismo. La reducción de los residuos es importante desde el punto de vista ambiental, ya que se verterían $5820 \mathrm{~kg} / \mathrm{h}$ menos de solvente (etanol: agua) al ambiente. La correcta disposición final de estos residuos (realizada mediante incineración) sería un costo adicional que se debe asumir. Los rendimientos del proceso para la extracción fueron calculados teniendo en cuenta $141,351 \mathrm{~kg} / \mathrm{h}$ de cáscara de cacao, obteniéndose $6,0 \%$ de Polifenoles totales y $56,80 \%$ fibra dietaría lo que indica una viabilidad en el aprovechamiento de los residuos de cáscara de cacao como nutrientes.

La viabilidad económica preliminar del proceso de extracción de polifenoles y fibra dietaría total a partir de los residuos de cáscara del cacao CCN51 se obtuvo con el simulador SuperPro Designer ${ }^{\circledR} \quad$ v.9.0 académica. Los datos obtenidos se presentan en la tabla IV, en la que se resumen los costos asociados con capital invertido, operación y producción. En dicha tabla se aprecia que el capital total invertido para la realización de la planta de extracción de polifenoles y fibra a partir del cacao CCN51 es de 2.106.470 dólares, con un costo de operación por año de 1.195.722 dólares. La producción por año es de 1200 Ton, produciéndose 65,88 Ton de polifenoles y 681,60 Ton de fibra. Bajo estas consideraciones el costo promedio de producción de un kilogramo de polifenoles sería de 18,15 USD; en lo que se refiere al costo asociado con la producción de la fibra total, éste fue de o USD $/ \mathrm{kg}$, lo anterior debido a que todos los costos fueron asociados con la producción de polifenoles y la fibra total fue obtenida como un producto secundario usando el mismo equipamiento y mano de obra que se empleó para la producción de los polifenoles totales. El precio de venta de PT se fijó en 48 dólares/kg y FD: 1 dólar/ $\mathrm{kg}$; siguiendo los valores aproximados del mercado según [6]. Los ingresos totales por año serían de 3.837 .024 dólares, con un margen bruto de $68,84 \%$ y un retorno de la inversión de $22,16 \%$, en un tiempo de recuperación de 5 años.

Tabla IV. Resumen preliminar de viabilidad económica de la planta extractora de PT y FD a partir de la cáscara del cacao CCN51.

\begin{tabular}{|c|c|c|}
\hline \multicolumn{2}{|c|}{ CONCEPTO } & DESCRIPCIÓN USD* \\
\hline \multicolumn{2}{|c|}{ Capital total invertido } & $2.106 .470,00$ \\
\hline \multicolumn{2}{|c|}{ Costo de operación } & $1.195 .722,00$ \\
\hline \multirow{2}{*}{ Producción } & Polifenoles & 74,30 Ton/año \\
\hline & Fibra & 693,70 Ton/año \\
\hline \multirow{2}{*}{ Costo unitario } & Polifenoles & $18,15 \mathrm{USD} / \mathrm{kg}$ \\
\hline & Fibra & $0,00 \mathrm{USD} / \mathrm{kg}$ \\
\hline \multicolumn{2}{|l|}{ Ingresos totales } & $3.837 .024,00$ \\
\hline \multicolumn{2}{|l|}{ Margen bruto } & $68,84 \%$ \\
\hline \multicolumn{2}{|c|}{ Retorno de la inversión } & $22,16 \%$ \\
\hline \multicolumn{2}{|c|}{ Tiempo de recuperación } & 5 AÑOS \\
\hline
\end{tabular}

\section{Conclusiones}

$122-820 \mathrm{X}$

SN 2422-5053

Se demostró que el software SuperPro Designer ${ }^{\circledR}$ v.9.0 académica constituye una 
No. 1

Enero - Junio 2017 ISSN 0122-820X E-ISSN 2422-5053 PP: $75-83$ herramienta de utilidad para el análisis de procesos en la industria cacaotera de la región. El modelo de simulación obtenido permite llevar a cabo estudios de escalamiento de producción y es una base indispensable para el mejoramiento del proceso de extracción de polifenoles y fibra.

Los resultados obtenidos muestran que es técnicamente factible construir una planta extractora de polifenoles, transformando 1200 Ton/año de residuos generados en el proceso de industrialización del cacao; para una operación continua de proceso que genera 74,30 toneladas año de polifenoles totales y 693,70 toneladas de fibra total.

En este tipo de proceso, materia prima utilizada y la tecnología empleada para la extracción, se genera un impacto positivo a nivel ambiental por la reutilización de solvente corriente S-115 con un (99\%) acorde con la tendencia tecnológica de producción más limpia y desarrollo sostenible.

\section{Agradecimientos}

Los autores agradecen la colaboración del Grupo de Ciencia y Tecnología de AlimentosCICTA de la Universidad Industrial de Santander.

\section{Referencias}

[1] L. Quintana, S. Gómez, A. García y N. Martínez, "Caracterización de tres índices de cosecha de cacao de los clones CCN51, ICS60 e ICS 95, en la montaña santandereana, Colombia”, Revista de alimentación agraría y ambiental, vol. 6, no. 1, pp. 253-265, 2015.

[2] A. Pallares-Pallares, J.A. PereaVillamil, L.J. López-Giraldo, "Impacto de las condiciones de beneficio sobre los compuestos precursores de aroma en granos de cacao (Theobroma cacao

L) del clon CCN-51", Respuestas, vol. 21, no. 1, pp. 120-133, 2016

[3] S. Gómez, L. Quintana, A. García and N. Martinez, "Clone sensory profile of cocoa (Theobroma cacao L.) CCN51 (first vintage 2015)".@LIMENTECH CIENCIA Y TECNOLOGÍA ALIMENTARIA, vol. 13, no. 1, pp. 6065, 2015.

[4] O. Ortiz, R. Villamizar and J. Rangel, "Applying life cycle management of colombian cocoa production", Food Science and Technology (Campinas), vol. 34, no. 1, pp. 62-68, 2014.

[5] L. Pérez y N. Pulido, "Determinación experimental y modelamiento de los perfiles de extracción supercrítica para la obtención de compuestos bioactivos a partir de cáscara de cacao (Clon CCN-51)"; Universidad Industrial de Santander: Bucaramanga, 2014.

[6] Trade Map - Lista de los países importadores para el producto seleccionado en 2015. Octubre 2016. [En línea]. Disponible en: http://www. trademap.org.

[7] L. Flórez y M. Jerez, "Implementación de un método enzimático-gravimétrico para la determinación de fibra dietaría soluble e insoluble en residuos de cacao"; Universidad Industrial de Santander: Bucaramanga, 2016.

[8] S. Toro, M.R. Estupiñan and L.J. López, "Cocoa husk as source of natural phenolic antioxidants: comparision of polyphenols and antioxidant activity in theobroma cacao beans and husk", I Congr. Int. de investigación e innovación en ingeniería, ciencia y tecnología de alimentos, Medellín, Fac.Nal.Arg, 2014 
[9] L. Sotelo, A. Alvis y G. Arrázola, "Evaluación de la epicatequina, teobromina y cafeína en cáscaras de cacao (Theobroma cacao L.), determinación de su capacidad antioxidante". Revista colombiana de ciencias hortícolas, vol 9, no. 1, pp. 124-134,2015.

[10] J. Garrido, H. Navarro, M. Diaz de los Rios y I. Perez, "Evaluación de alternativas de producción de levadura forrajera a partir de vinazas mediante simulación con SuperPro Designer sobre derivados de la caña de azúcar". ICIDCA, vol. 44, no. 2, pp. 34-40, 2010.

[11] S. Villamizar y S. Barragan, Análisis preliminar de la simulación y factibilidad económica del proceso de recuperación de polifenoles a partir de cascara de cacao; Universidad Industrial de Santander: Bucaramanga, 2014.

[12] J. Godoy, Obtención de antioxidantes a partir de grano de cacao: condiciones favorables de inactivación de la enzima Polifenol Oxidasa, extracción de polifenoles y ajuste de parámetros cinéticos; Universidad Industrial de Santander: Bucaramanga, 2015.

[13] D. Abarca, R. Martínez, J. Muñoz, M. Torres y G. Vargas, "Residuos de café, cacao y cladodio de tuna: Fuentes promisorias de fibra dietaria", Revista Tecnológica-ESPOL, vol. 23, no. 2, 2010.

[14] O. Carreño y L. Rojas, Determinación y ajuste de parámetros cinéticos de la extracción de polifenoles totales a partir de la cascara de cacao; Universidad Industrial de Santander: Bucaramanga, 2014. 